\title{
Surface polar optical phonon interaction induced many-body effects and hot-electron relaxation in graphene
}

\author{
E. H. Hwang ${ }^{1,2}$ and S. Das Sarma ${ }^{1}$ \\ ${ }^{1}$ Condensed Matter Theory Center, Department of Physics, \\ University of Maryland, College Park, Maryland 20742-4111 and \\ ${ }^{2}$ SKKU Advanced Institute of Nanotechnology, Sungkyunkwan University, Suwon 440-746, Korea
}

(Dated: October 3, 2018)

\begin{abstract}
We theoretically study various aspects of the electron-surface optical phonon interaction effects in graphene on a substrate made of polar materials. We calculate the electron self-energy in the presence of the surface phonon-mediated electron-electron interaction focusing on how the linear chiral graphene dispersion is renormalized by the surface phonons. The electron self-energy as well as the quasiparticle spectral function in graphene are calculated, taking into account electron-polar optical phonon interaction by using a many body perturbative formalism. The scattering rate of free electrons due to polar interaction with surface optical phonons in a dielectric substrate is calculated as a function of the electron energy, temperatures, and carrier density. Effects of screening on the self-energy and scattering rate are discussed. Our theory provides a comprehensive quantitative (and qualitative) picture for surface phonon interaction induced many-body effects and hot electron relaxation in Dirac materials.
\end{abstract}

\section{INTRODUCTION}

Lattice vibrations are inevitable intrinsic sources of carrier scattering in semiconductors and often dominate transport near room temperature ${ }^{1,2}$. In general, scattering by acoustic phonons is the main contribution to the electronic resistivity at low temperatures, and the longitudinal-optical (LO) phonons in polar materials limit the room temperature mobility through their coupling with carriers via the long-range Fröhlich interaction $^{3}$. Since graphene is made of carbons it is a non-polar material and the lack of strong long-range polar optical phonon scattering leads to very high intrinsic (i.e., phonon-limited) room-temperature graphene mobility ${ }^{4-10}$ and indeed the room-temperature graphene mobility is typically very high in disorder-free suspended graphene samples 11,12 . The weak deformation potential scattering from the thermal lattice acoustic phonons mainly limits the intrinsic mobility of graphene at room temperature and the acoustic phonon scattering gives a quantitatively small contribution even at room temperature due to the high Fermi temperature at high carrier density (and the small deformation potential coupling) of graphene $e^{8-10}$.

However, most current available graphene samples for fundamental studies and certainly for technological applications use a polar substrate such as $\mathrm{SiO}_{2}, \mathrm{SiC}$, or $\mathrm{HfO}_{2}$. Such polar substrates allow for the existence of polar optical phonons localized near the graphene-substrate interface which could be an important scattering source for graphene carriers through the long-range Fröhlich coupling. The general eigenvalue equation for a surface phonon mode has been derived at a planar interface between two polar dielectrics ${ }^{13-15}$. Since the surface optical (SO) phonon is a well-defined surface property at the interface of the polar semiconductors 15,16 it is possible that carriers in graphene layer couple to the SO phonon of the underlying substrate lattice via the long- range polar Fröhlich coupling. Recently, the possible role of surface optical phonon on high temperature graphene mobility has been pointed out ${ }^{5-7}$. Due to the polar nature of the gate dielectrics used in graphene field effect transistors, the carriers in the conducting channel electrically couple to the long-range surface phonons created at the dielectric interface. In general, the surface optical phonon contributes little to the carrier resistivity at low temperatures due to its high energy $\left(\omega_{S O P} \gtrsim 50 \mathrm{meV}\right)$. However, the contributions of other scattering processes to the graphene resistivity are small at room temperature and therefore the SO phonon may become the dominant scattering mechanism in graphene on a polar dielectric substrate at high temperatures. It is therefore possible, perhaps even likely, that in the context of graphene technological applications, SO phonon effects are particularly important and must be taken into account. In addition, in a recent experiment ${ }^{17-20}$, the strongly coupled plasmon-phonon mode dispersion has been measured by the angle-resolved reflection electronenergy-loss spectroscopy and it is found that the electronphonon coupling give rise to the many interesting manybody effects in graphene on a polar dielectric substrate ${ }^{21}$. This again points to the possible importance of carrierSO phonon coupling in determining the electronic properties of graphene on a substrate.

Since the electron-optical phonon interaction leads to many-body renormalization of the single-particle free carrier properties ${ }^{22,23}$ while at the same affecting the transport properties ${ }^{5,7}$ a good understanding of electronSO phonon coupling is thus important in developing quantitative theories for many different experimental studies in graphene. In this paper we calculate the electron self-energy and spectral function of graphene on a polar substrate such as $\mathrm{SiO}_{2}, \mathrm{SiC}$, or $\mathrm{HfO}_{2}$. Our goal in this paper is to provide a fairly detailed theoretical picture for the electron-SO phonon interaction induced polar scattering of free carriers. Many electronic properties of 
graphene on polar substrates are expected to be modified by electron-SO phonon scattering via the Flöhlich interaction. On a microscopic level this interaction renormalize the Fermi velocity of graphene. A thorough quantitative understanding of the electron-SO phonon interaction induced many-body effects in high mobility graphene is very important with respect to both the device operation and the fundamental physics of graphene on substrates. This surface polaronic (i.e. interaction with SO phonons) effect is an important factor in determining the high temperature mobility and the hot electron properties of graphene on substrates.

In this paper, we focus on the effect of electron-SO phonon interaction effects on the real, $\operatorname{Re}[\Sigma]$, and the imaginary part, $\operatorname{Im}[\Sigma]$, of electron self-energy. From $\operatorname{Im}[\Sigma]$, we can extract the quasiparticle scattering rate (scattering lifetime), which gives information relevant to possible applications, such as the hot electron relaxation rate and the energy dissipation rate of injected carriers in a graphene-based device. We calculate the scattering rate as a functions of wave vector, energy, density and temperature and discuss the role of screening effects. Since the electron- surface polar optical phonon interaction has a long range nature the consideration of screening effects is crucial in order to understand quantitative physical properties of graphene. We find that the scattering rates with screened interaction is reduced by a factor of five, compared with the results with unscreened interaction. Our consideration of screening (i.e., the static random phase approximation (RPA) screening) may overestimate the screening effect on scattering rate at low temperatures, but it provides an upper limit of the screening effects. In addition to calculating the properties of $\operatorname{Im}[\Sigma]$ due to SO phonon interaction, we also calculate the $\operatorname{Re}[\Sigma]$, which gives us the corresponding graphene velocity renormalization due to the surface polaronic effect. We also provide results for the graphene spectral function due to electron-SO phonon scattering, which can be directly observed in scatting tunneling spectroscopy (STS) and angle-resolved photo-electron spectroscopy (ARPES). Our theory and results are directly relevant to the electronic properties of doped or gated graphene on polar substrates where SO polaron effects may very well be significant.

The paper is organized as follows. In Sec. II the generalized theory is presented to calculate the self-energy of electron in the presence of electron-SO phonon interaction. Sec. III presents the results of self-energy and spectral function. We also show the scattering rate as a function of energy, momentum, density, and temperature. We summarize in Sec. IV with a discussion.

\section{THEORY}

The Hamiltonian of graphene is well-approximated by a 2D Dirac equation for massless particles, $H_{0}=v_{F} \sigma \cdot \boldsymbol{k}$, where $v_{F}$ is the Fermi velocity of graphene, $\sigma$ is the pseo- dospin $2 \times 2$ Pauli matrices, and $\boldsymbol{k}$ is the $2 \mathrm{D}$ momentum relative to the Dirac points (we use $\hbar=1$ throughout this paper). The two components of the spinors correspond to occupancy of the two sublattices of the honeycomb structure in a hexagonal lattice. This $H_{0}$ gives a linear energy dispersion relation $\varepsilon_{\boldsymbol{k}, s}=s v_{F}|\boldsymbol{k}|$, where $s=+1(-1)$ for the conduction (valence) band. The corresponding density of states (DOS) is $D(\varepsilon)=g_{s} g_{v}|\varepsilon| /\left(2 \pi v_{F}^{2}\right)$, where $g_{s}=2, g_{v}=2$ are the spin and valley degeneracies, respectively.

The central quantity we calculate in this paper is the leading order electronic self-energy correction due to electron-surface optical (SO) phonon interaction via Fröhlich polar coupling ${ }^{15}$. In the presence of the long range electron-SO phonon coupling, electrons interact among themselves through the Coulomb interaction and through virtual-SO phonon exchange via the Fröhlich interaction. The electron-SO phonon interaction is given by

$$
H_{e-p h}=\sum_{k q} \sum_{s s^{\prime}} M_{k q}^{s s^{\prime}} c_{k+q s^{\prime}}^{\dagger} c_{k s}\left(b_{q}+b_{-q}^{\dagger}\right)
$$

where $c_{k s}^{\dagger}$ is the electron $(s=+1)$ or hole $(s=-1)$ creation operator, $b_{q}^{\dagger}$ and $b_{q}$ are creation and destruction operators of surface optical phonon, and the interaction matrix element $M_{s}^{s s^{\prime}}$ is defined by

$$
M_{k q}^{s s^{\prime}}=M(q) F_{s k+q}^{\dagger} F_{s^{\prime} k},
$$

where $F_{s k}$ is the chiral spinor and given by

$$
F_{s k}=\frac{1}{\sqrt{2}}\left(\begin{array}{c}
s \\
e^{i \theta_{k}}
\end{array}\right)
$$

with $s= \pm 1, \theta_{k}=\tan ^{-1}\left(k_{y} / k_{x}\right)$ is the angle between $\boldsymbol{k}, \boldsymbol{k}^{\prime}$, arises from the overlap of $|s \boldsymbol{k}\rangle$ and $\left|s^{\prime} \boldsymbol{k}^{\prime}\right\rangle \stackrel{2}{\underline{2}}$ The strength of the coupling $M(q)$ is given by

$$
[M(q)]^{2}=\frac{M_{0}^{2}}{q \epsilon(q)} e^{-2 q d}
$$

where

$$
M_{0}^{2}=\pi e^{2} \omega_{S O}\left[\frac{1}{\epsilon_{\infty}+1}-\frac{1}{\epsilon_{0}+1}\right],
$$

and $\epsilon(q)$ is the dielectric (screening) function of graphene ${ }^{24}, d$ is the separation distance between graphene layer and substrate, $\omega_{S O}$ is the surface optical phonon frequency, and $\epsilon_{0}\left(\epsilon_{\infty}\right)$ is the static (high frequency) dielectric constant. In Eq. (4), we set $\epsilon(q)=1$ for the unscreened case. For screened interaction we use the static RPA dielectric function appropriate for graphene, which is give by

$$
\epsilon(q)=1+v(q) \Pi(q)
$$

where $v(q)=2 \pi e^{2} / \kappa q$ is the electron-electron Coulomb potential with the effective background lattice dielectric constant $\kappa=\left(\epsilon_{0}+1\right) / 2$, and $\Pi(q)=\Pi(q, T)$ is 
the electronic polarizability function of graphene which depends on both wave vector and temperature. For isotropic media the frequency of SO phonons $\omega_{S O}$ is related to the transverse optical (TO) bulk phonon $\omega_{T O}$ as $\omega_{S O} / \omega_{T O}=\sqrt{\left(\epsilon_{0}+1\right) /\left(\epsilon_{\infty}+1\right)} \cdot 13,15$ Note that the bulk longitudinal optical phonons $\omega_{L O}$ and $\omega_{T O}$ are connected with the dielectric constants by the Lyddane-Sachs-Teller relation ${ }^{3} \omega_{L O} / \omega_{T O}=\sqrt{\epsilon_{0} / \epsilon_{\infty}}$.

The SO-phonon mediated electron-electron effective interaction (i.e. in the leading-order screened Fröhlich coupling) is given by

$$
v_{p h}^{s s^{\prime}}(q, \omega)=\left[M_{0}(q)\right]^{2} D_{0}(\omega) F_{k k^{\prime}}^{s s^{\prime}}
$$

which depends on both wave vector and frequency. In Eq. (7) $D_{0}(\omega)$ is the unperturbed SO-phonon propagator and given by

$$
D_{0}(\omega)=\frac{2 \omega_{S O}}{\omega^{2}-\omega_{S O}^{2}}
$$

and

$$
F_{k k^{\prime}}^{s s^{\prime}}=\left|F_{s k}^{\dagger} F_{s^{\prime} k+q}\right|^{2}=\left[1+s s^{\prime} \cos \left(\theta_{k k^{\prime}}\right)\right] / 2
$$

The self-energy $\Sigma$ within the leading-order approximation is give $\mathrm{by}^{3}{ }^{3}$

$\Sigma_{s}\left(\boldsymbol{k}, i \omega_{n}\right)=-\frac{1}{\beta} \sum_{s^{\prime}} \sum_{\boldsymbol{q}, i \nu_{n}}\left[M_{k q}^{s s^{\prime}}\right]^{2} D\left(q, i \omega_{n}\right) G_{0, s^{\prime}}\left(\boldsymbol{k}+\boldsymbol{q}, i \omega_{n}+i \nu_{n}\right)$.

Here, $\beta=1 / k_{B} T, s, s^{\prime}= \pm 1$ are band indices, $G_{0, s}\left(\mathbf{k}, i \omega_{n}\right)=1 /\left(i \omega_{n}-\xi_{\mathbf{k} s}\right)$ is the bare Green's function, $\omega_{n}, \nu_{n}$ are Matsubara fermion frequencies, and $M_{k q}^{s s^{\prime}}$ the interaction matrix element. After the standard analytical continuation from $i \omega_{n}$ to $\omega+i 0^{+}$the retarded self-energy $\Sigma^{\text {ret }}$ is obtained,

$$
\Sigma_{s}^{\mathrm{ret}}(\boldsymbol{k}, \omega)=\sum_{s^{\prime}} \int \frac{d \boldsymbol{q}}{(2 \pi)^{2}} \frac{M_{0}^{2} e^{-2 q d}}{q \epsilon(q)} F_{s s^{\prime}}(k, k-q)\left[\frac{N_{0}+n_{F}\left(k_{s^{\prime}}\right)}{\omega+i \eta+\omega_{S O}-\xi_{k^{\prime} s^{\prime}}}+\frac{N_{0}+1-n_{F}\left(k_{s^{\prime}}\right)}{\omega+i \eta-\omega_{S O}-\xi_{k^{\prime} s^{\prime}}}\right]
$$

where $\xi_{\boldsymbol{k}, s}=v_{F}|k|-\mu$, is the electron energy relative to the chemical potential $\mu\left(=E_{F}\right.$ at $\left.T=0\right)$. The function $N_{0}$ and $n_{F}$ are Bose and Fermi distribution function defined by

$$
N_{0}=\frac{1}{e^{\beta \omega_{S O}}-1}
$$

and

$$
n_{F}\left(k_{s}\right)=\frac{1}{e^{\beta \xi_{k s}}+1},
$$

where the chemical potential $\mu$ at finite temperature is determined from the total electron density $n$ of the system. The self energy is an explicit function of the independent variable momentum $(k)$ and energy $\omega$. We obtain the real and imaginary parts of the electronic self energy

$$
\operatorname{Re}\left[\Sigma_{s}(\boldsymbol{k}, \omega)\right]=M_{0}^{2} \sum_{s^{\prime}} \int \frac{k^{\prime} d k^{\prime}}{(2 \pi)^{2}} G_{s s^{\prime}}\left(\boldsymbol{k}, \boldsymbol{k}^{\prime}\right)\left[\frac{N_{0}+n_{F}\left(\xi_{k^{\prime} s^{\prime}}\right)}{\omega+\omega_{S O}-\xi_{k^{\prime} s^{\prime}}}+\frac{N_{0}+1-n_{F}\left(\xi_{k^{\prime} s^{\prime}}\right)}{\omega-\omega_{S O}-\xi_{k^{\prime} s^{\prime}}}\right]
$$

$\left.\operatorname{Im}\left[\Sigma_{s}^{(} \boldsymbol{k}, \omega\right)\right]=-\pi M_{0}^{2} \sum_{s^{\prime}} \int \frac{k^{\prime} d k^{\prime}}{(2 \pi)^{2}} G_{s s^{\prime}}\left(\boldsymbol{k}, \boldsymbol{k}^{\prime}\right)\left[\left(N_{0}+n_{F}\left(\xi_{k^{\prime} s^{\prime}}\right)\right) \delta\left(\omega+\omega_{S O}-\xi_{k^{\prime} s^{\prime}}\right)+\left(N_{0}+1-n_{F}\left(\xi_{k^{\prime} s^{\prime}}\right)\right) \delta\left(\omega-\omega_{S O}-\xi_{k^{\prime} s^{\prime}}\right)\right]$

where

$$
G_{s s^{\prime}}\left(\boldsymbol{k}, \boldsymbol{k}^{\prime}\right)=\int d \theta \frac{e^{-2\left|\boldsymbol{k}-\boldsymbol{k}^{\prime}\right| d}}{\left|\boldsymbol{k}-\boldsymbol{k}^{\prime}\right| \epsilon\left(\left|\boldsymbol{k}-\boldsymbol{k}^{\prime}\right|\right)} F_{s s^{\prime}}\left(\boldsymbol{k}, \boldsymbol{k}^{\prime}\right)
$$

where $\theta$ is the angle between $\boldsymbol{k}$ and $\boldsymbol{k}^{\prime}$.

For $T=0$ the imaginary part of the self energy, Eq. (15), becomes 


$$
\begin{aligned}
\operatorname{Im}\left[\Sigma_{s}(k, \omega)\right]=A \sum_{s^{\prime}= \pm 1}\left\{G_{s s^{\prime}}\left[k, s^{\prime}\left(\omega+\omega_{S O}+E_{F}\right)\right] s^{\prime}\left(\omega+\omega_{S O}+E_{F}\right) \theta\left(-\omega-\omega_{0}\right) \theta\left[s^{\prime}\left(\omega+\omega_{S O}+E_{F}\right)\right]\right. \\
\left.+G_{s s^{\prime}}\left[k, s^{\prime}\left(\omega-\omega_{S O}+E_{F}\right)\right] s^{\prime}\left(\omega-\omega_{S O}+E_{F}\right) \theta\left(\omega-\omega_{0}\right) \theta\left[s^{\prime}\left(\omega-\omega_{S O}+E_{F}\right)\right]\right\}
\end{aligned}
$$

where $A=-\pi M_{0}^{2} /(2 \pi)^{2} v_{F}$ and $\theta(x)=1$ for $x \geq 0$ and 0 for $x<0$. The first term in Eq. (17) denotes SO phonon absorption by the electron, whereas the second term denotes the emission of SO phonon. From Eq. (17) we find that the phonon emission is only allowed for $\omega>\omega_{S O}$, and the phonon absorption for $\omega<-\omega_{S O}$. Thus, due to the phase space restriction there is a region where $\operatorname{Im}\left[\Sigma\left(k_{F}, \omega\right)\right]$ becomes zero for energies within $\omega_{S O}$ of the Fermi energy, i.e., for $-\omega_{S O}<\omega<\omega_{S O}$ $\operatorname{Im}\left[\Sigma\left(k_{F}, \omega\right)\right]=0$. Note that $\omega=0$ corresponds to the Fermi energy. The vanishing of $\operatorname{Im}[\Sigma]$ indicates that a quasiparticle within this region cannot decay by emitting (or absorbing) surface phonons because there is no available final state to decay. A quasiparticle with an initial state energy $\omega$ has a final state energy $\omega-\omega_{S O}$ by emitting an SO phonon. The final state energy of the electron must greater than the Fermi energy (i.e., $\omega-\omega_{S O}>0$ ) for a decay process to occur. Otherwise the transition cannot occur because the final states are all occupied. Thus, for the quasiparticle with energy $\omega<\omega_{S O}, \operatorname{Im}[\Sigma]=0$. An electron outside this energy range can lose energy by emitting SO phonons. For a quasiparticle with the initial energy $\omega$ has the final state $\omega+\omega_{S O}$ by absorbing an SO phonon. Thus, the quasiparticle with the initial energy $-\omega_{S O}<\omega$ cannot decay by absorbing an SO phonon. Thus, for $-\omega_{S O}<\omega<\omega_{S O}, \operatorname{Im}\left[\Sigma\left(k_{F}, \omega\right)\right]=0$. In addition to this forbidden region, the decay of an electron to the Dirac point is not allowed because the DOS is zero at that point, which occurs at $\omega=-E_{F}-\omega_{S O}$ (i.e., the quasiparticle with the initial energy $-E_{F}-\omega_{S O}$ cannot decay to the final state energy $-E_{F}$ (i.e. Dirac point) after absorbing an SO phonon). However, we note that this forbidden gap exists only for the self-energy contribution from surface optical phonons and at zero temperature. The quasiparticle will have finite values of $\operatorname{Im}[\Sigma]$ in this energy region arising from scattering by impurities and from electron-electron interactions.

From the self energy, $\Sigma(\boldsymbol{k}, \omega)$, we can obtain the singleparticle spectral function

$$
A(k, \omega)=-2 \operatorname{Im} G(k, \omega)
$$

where

$$
[G(k, \omega)]^{-1}=\left[G_{0}(k, \omega)\right]^{-1}-\Sigma(k, \omega)
$$

Thus, we have

$$
A(k, \omega)=-\frac{2 \operatorname{Im} \Sigma(k, \omega)}{\left[\omega-\xi_{k}-\operatorname{Re} \Sigma(k, \omega)\right]^{2}+[\operatorname{Im} \Sigma(k, \omega)]^{2}} .
$$

The spectral function $A(k, \omega)$ can roughly be interpreted as the probability density of the different energy eigenstates required to make up a specific $k$ state. The spectral function shows a sharp peak at the quasiparticle state and becomes a $\delta$ function when $\operatorname{Im}[\Sigma]=0$. The spectral function must satisfy the sum rule

$$
\int \frac{d \omega}{2 \pi} A(k, \omega)=1
$$

and in all our numerical calculations we have explicitly checked that this sum rule is generally satisfied to within less than a percent.

The inverse quasiparticle lifetime (or, equivalently, the scattering rate) $\Gamma_{s}(\boldsymbol{k})$ of state $|s \boldsymbol{k}\rangle$ is obtained by setting the frequency in imaginary part of the self-energy to the on-shell (bare quasiparticle) energy $\xi_{s \boldsymbol{k}}=s v_{F}|\boldsymbol{k}|-E_{F}$, i.e.

$$
\Gamma_{s}(\boldsymbol{k})=2 \operatorname{Im}\left[\Sigma_{s}^{\mathrm{ret}}\left(\boldsymbol{k}, \xi_{\boldsymbol{k} s}\right)\right] .
$$

The self-energy approximation used here is equivalent to the generalized Born approximation for the scattering rate. Note that the integrand of Eq. (15) is non-zero only when $\xi_{k s}= \pm \omega_{S O}+\xi_{k^{\prime} s^{\prime}}$, which correspond to emission (+) and absorption (-) of surface optical phonon, respectively.

\section{RESULTS}

In this section we present the results for graphene on SiC. The parameters used in this calculation are following: the surface optical phonon frequency $\omega_{S O}=117.6$ $\mathrm{meV}$, the longitudinal optical phonon frequency, $\omega_{L O}=$ $120.7 \mathrm{meV}$, the static (high frequency) dielectric constant $\epsilon_{0}=9.72\left(\epsilon_{\infty}=6.52\right)$.

In Fig. 1 we show the calculated self-energy as a function of energy $\left(\omega / E_{F}\right)$ for $k=k_{F}, d=0$, and $T=0$. Note that $\omega=0$ and $-E_{F}$ represent the Fermi energy and Dirac point, respectively. The density $n=2 \times 10^{12} \mathrm{~cm}^{-2}$ is used in the calculation, which corresponds to $E_{F}=165$ meV. The Fermi momentum $\left(k_{F}\right)$ and the Fermi energy $\left(E_{F}\right.$, relative to the Dirac point energy) of graphene are given by $k_{F}=\left(4 \pi n / g_{s} g_{v}\right)^{1 / 2}$ and $\left|E_{F}\right|=v_{F} k_{F}$ where $n$ is the $2 \mathrm{D}$ carrier (electron or hole) density. $\Sigma_{+}\left(\Sigma_{-}\right)$represents the self energy for $s=1(s=-1)$. In Fig. 1 (a) and (b) the self-energies are calculated without screening effect by putting $\epsilon(q)=1$ in Eq. (11), but in (c) and (d) the RPA screening function is used to calculate the self-energies. As discussed in Sec. II, $\operatorname{Im}\left[\Sigma_{s}\right]$ vanishes for $-\omega_{S O}<\omega<\omega_{S O}$ and at $\omega=-E_{F}-\omega_{S O}$, and the range of the forbidden gap is independent of screening. For $s=-1$ there is a sharp strong peak in $\operatorname{Im}\left[\Sigma_{-}\right]$. This singular behavior for unscreened self energy for $s=-1$ is unphysical and arises from the long range nature of 

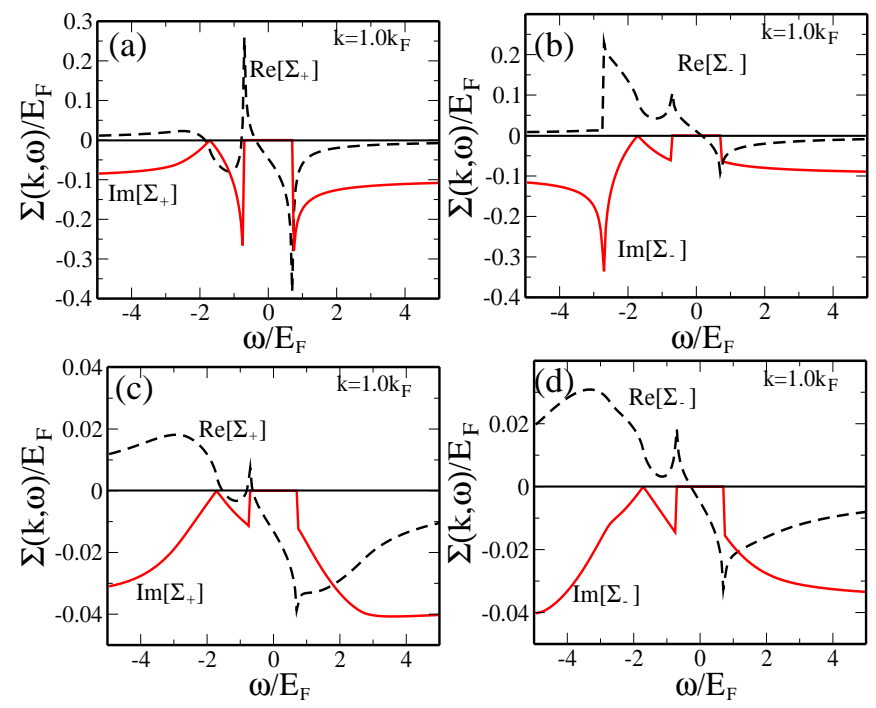

FIG. 1. Calculated self energies as a function of energy, $\omega / E_{F}$. In (a) and (b) $\Sigma_{ \pm}(k, \omega)$ is calculated without screening effect [i.e. $\epsilon(q)=1$ in Eq. (11)] and for $k=k_{F}, d=0$, and $T=0$. In (c) and (d) the self-energy $\Sigma_{ \pm}(k, \omega)$ is calculated with RPA screening function. Solid red (dashed black) lines indicate imaginary (real) parts of the self energy.

the electron- SO phonon interaction, i.e., $v_{p h} \propto 1 / q$. The RPA screening makes two significant modifications to the self energy compared with the results with the unscreened electron-SO phonon interaction. The screening effects reduce the magnitude of both $\operatorname{Re}[\Sigma]$ and $\operatorname{Im}[\Sigma]$ by roughly a factor of 10 , and removes the logarithmic singularity in $\operatorname{Im} \Sigma_{-}$occurring at $\omega=-E_{F}-\omega_{S O}-v_{F} k$. Since screening makes the long range electron-phonon interaction finite, the singularity at $q=0$ (or, $k^{\prime}=k$ ) is removed by changing $q$ to $q \epsilon(q)$ in Eq. (17).

In Fig. $2 \operatorname{Im}\left[\Sigma_{ \pm}(k, \omega)\right]$ is shown as a function of energy $\omega$ for $k=k_{F}$ and $T=0$, and for different values of $d=0,1$, and $2 \mathrm{~nm}$, where $d$ is the distance between graphene and the interface of the dielectric substrate. In Fig. 2(a)(b) the self-energy is calculated without screening effects and in (c) (d) the RPA screening in electronphonon interaction is considered. As the distance $d$ increases the magnitude of the $\operatorname{Im}[\Sigma]$ decreases due to the exponential dependence of the interaction, i.e., $e^{-2 q d}$ in the electron-SO phonon interaction, [see Eqs. (44) and (77)]. In Fig. 2(a) and (b) the logarithmically diverging peaks at $\omega= \pm \omega_{S O}$ and $\omega=-2 k_{F}+\omega_{S O}$ arises from the singular behavior of the long range electron-SO phonon interaction at $q=0$. However, as discussed in Fig. 1, this peaks disappear in the presence of screening as shown in Fig. 2 (c) and (d). For comparison of these results with the imaginary parts of the self-energy calculated with non-polar in-plane LO phonon $\left(\omega_{L O} \sim 200\right.$ $\mathrm{meV}$ ) arising from the lattice vibration of graphene itself which has been discussed in Ref. [22] and [25], we consider the self-energy for $d=0$ and the unscreened case $(\epsilon(q)=1)$. Then, the main difference between these two
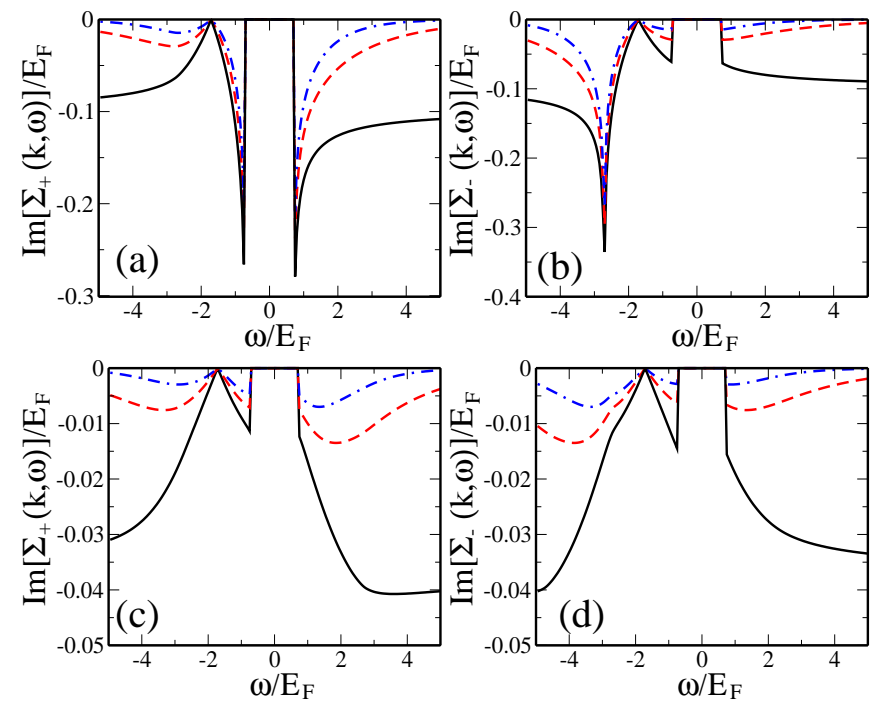

FIG. 2. Imaginary part of the self energy as a function of energy for $k=k_{F}$ and $T=0$ and for different values of distance between graphene and the dielectric substrate, $d=0$ (solid line), 1nm (dashed line), 2nm (dot-dashed line) (a),(b) are calculated without screening effect and (c),(d) with RPA screening function.

cases appears in Eq. (17). For non-polar LO phonon we have $G_{s s^{\prime}}=\pi$, and for polar SO phonon we have

$$
\begin{aligned}
G_{s, s}\left(k, k^{\prime}\right) & =\frac{k+k^{\prime}}{k k^{\prime}}[K(r)-E(r)] \\
G_{s,-s}\left(k, k^{\prime}\right) & =\frac{k+k^{\prime}}{k k^{\prime}}\left[-\left(\frac{k-k^{\prime}}{k+k^{\prime}}\right)^{2} K(r)+E(r)\right](23)
\end{aligned}
$$

where $K(r)$ and $E(r)$ are the complete elliptic integral of the first and second kinds, respectively, and $r=2 k k^{\prime} /\left(k^{2}+k^{\prime 2}\right)$. For the non-polar LO phonon $\operatorname{Im}[\Sigma]$ increases linearly with $|\omega|$ for larger $|\omega|$ because the DOS increases linearly with $|\omega|$. For polar SO phonon the long range interaction and the linear energy dispersion of graphene DOS compensate each other and the cancellation of these two effects makes the self energy saturate for large $|\omega|$. The emission (or absorption) of SO phonon does not depend on the quasiparticle energy for large $|\omega|$ (our numerical results show that the $\operatorname{Im}[\Sigma]$ saturates for $\left.|\omega| \gtrsim 4 E_{F}\right)$. Thus, high energy hot electrons decay more effectively by emitting (absorbing) graphene LO phonons than low energy electron. However, for SO phonons the damping of the electron by emitting (absorbing) SO phonons depends weakly on the energy of electrons. For finite $d, \operatorname{Im}[\Sigma]$ decreases faster than for $d=0$ as $|\omega|$ increases, then the high energy hot electrons do not decay effectively by emitting (or absorbing) remote $\mathrm{SO}$ phonons.

In Figs. 3 6 we show the calculated self-energy $\Sigma(k, \omega)$ and the spectral function $A(k, \omega)$ for different values of $k=0.51 .0$, and $1.5 k_{F}$. Figs. 3 and 4 show the results calculated with unscreened electron-SO phonon interaction for $s=+1$ (conduction band) and $s=-1$ 

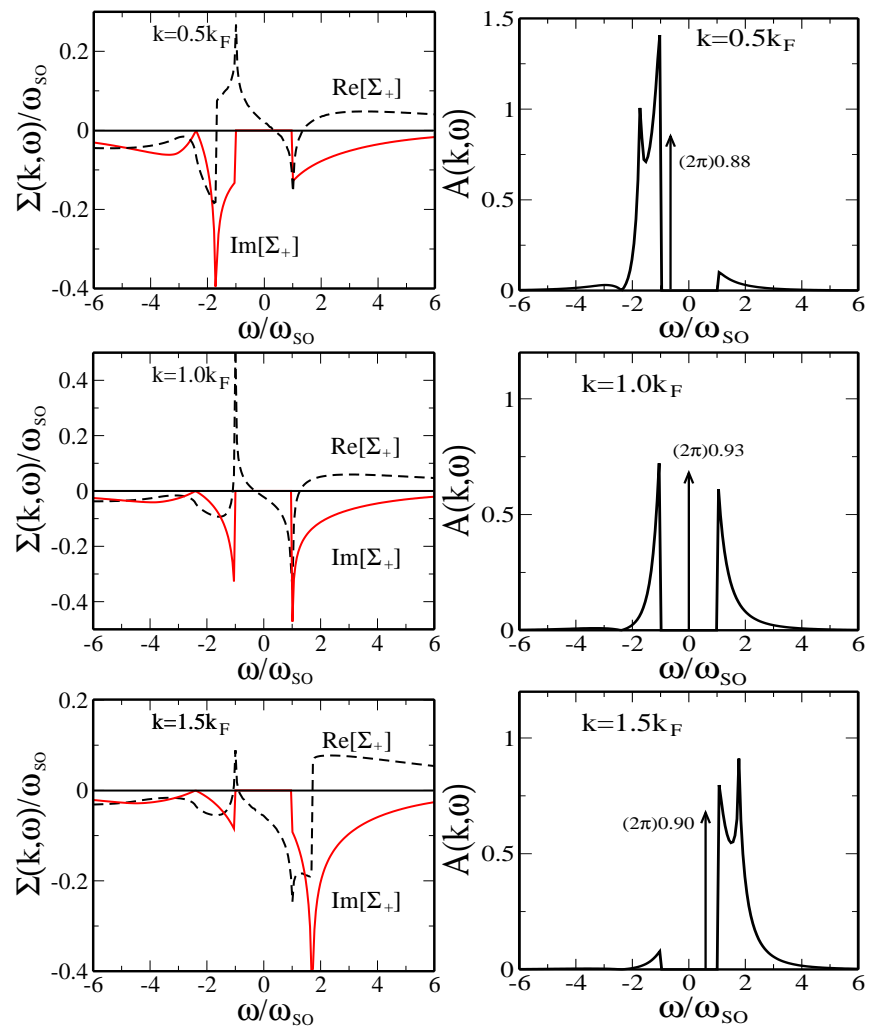

FIG. 3. Calculated self energy $\Sigma_{+}$and spectral function $A_{+}$ as a function of energy for different wave vectors, $k=0.5$, 1.0, $1.5 k_{F}$ and for $d=1 \mathrm{~nm}$ and $T=0$. Note that the energies are measured in terms of $\omega_{S O}$ instead of $E_{F}$. Screening effect is not considered, $\epsilon(q)=1$. The vertical arrows indicate $\delta$-function peaks at the quasiparticle energy with the spectral weights shown next to the arrows. They are located at $\omega=-0.447,0.0$, and $0.454 E_{F}$ for $k=0.5,1.0,1.5 k_{F}$, respectively. We find that from the calculated spectral function the renormalized velocity due to the electron-SO phonon interaction is reduced, i.e., $v_{F}^{*}=0.88 v_{F}$ approximately for these results.

(valence band), respectively. The bare electron (hole) energy, $E(k)= \pm v_{F}|k|$, is modified due to the electron$\mathrm{SO}$ phonon interaction and the energy dispersion is given by the solution to Dyson's equation for a given $k$,

$$
\omega=\xi_{k}+\operatorname{Re}\left[\Sigma_{s}(k, \omega)\right]-\mu,
$$

where $\xi_{k}=v_{F}|k|-E_{F}$ and $\mu=\Sigma_{+}\left(k_{F}, 0\right)$ is the chemical potential. A peak in the spectral function corresponds to the solution to Dyson's equation and the quasiparticle energy for a given $k$. For $s=+1$ (Fig. 3) the spectral function has a well defined $\delta$-function peak as long as the peak is located inside the forbidden region. The vertical arrows in Fig. 3 indicate $\delta$-function peaks at the quasiparticle energy with the spectral weights shown next to the arrows. They are located at $\omega=-0.447,0.0$, and 0.454 $E_{F}$ for $k=0.5,1.0,1.5 k_{F}$, respectively, and these quasiparticle peaks consume almost $90 \%$ of the total spectral weight. The remaining part of the spectral weight goes to the incoherent background. We find that from the cal-
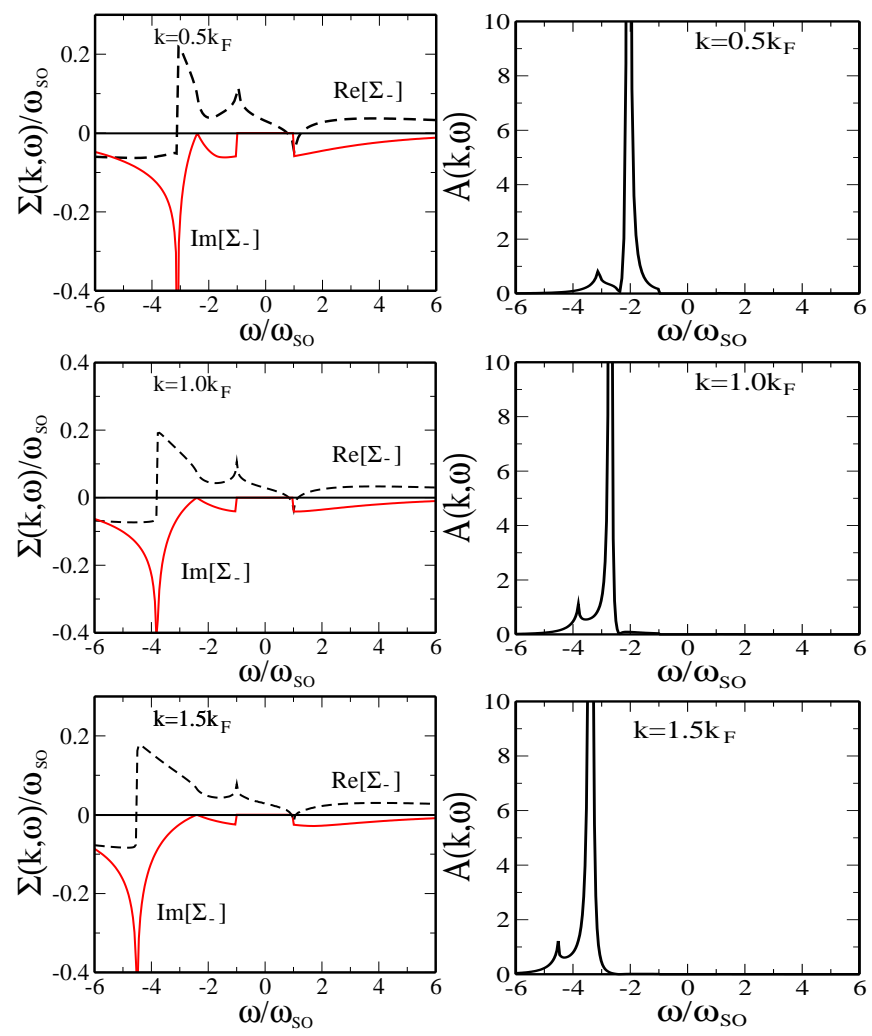

FIG. 4. Self energy $\Sigma$ and spectral function $A$ for $s=-1$ calculated with unscreened electron-SO phonon interaction. $\Sigma_{-}$and $A_{-}$are shown as a function of energy $\omega / \omega_{S O}$ for different wave vectors, $k=0.5,1.0,1.5 k_{F}$ and for $d=1$ $\mathrm{nm}$ and $T=0$. The quasiparticle peaks are not coherent for $s=-1$ and the peaks are broadened by emitting or absorbing SO phonons.

culated spectral function the renormalized velocity due to the electron-SO phonon interaction is reduced, i.e., $v_{F}^{*}=0.88 v_{F}$ approximately for these results. In Fig. 4 we show the self-energy $\Sigma_{-}(k, \omega)$ and the spectral function $A_{-}(k, \omega)$ for $s=-1$ and for different values of $k=0.5$ 1.0 , and $1.5 k_{F}$. For $s=-1$ the quasiparticle peaks are located outside the forbidden region and they decay by emitting (or absorbing) SO phonons. As a consequence, the spectral function has a peak with finite broadening instead of the $\delta$-function peak. The quasiparticle peaks for $s=-1$ (or in the valence band) are not coherent and the peaks are broadened by emitting or absorbing SO phonons.

Figs. [5] and 6] show the results calculated with the screened electron-SO phonon interaction for $s=+1$ (conduction band) and $s=-1$ (valence band), respectively. For $s=+1$ we have the $\delta$ function peak in the spectral function corresponding to the solution to Dyson's equation and the quasiparticle energy for a given $k$. However, the energy of the quasiparticle (i.e., the location of the peak) for a given wave vector is almost identical to that of the non-interaction systems. The peaks are located at $\omega=-0.49,0.0$, and $0.49 E_{F}$ for $k=0.5,1.0,1.5 k_{F}$, re- 

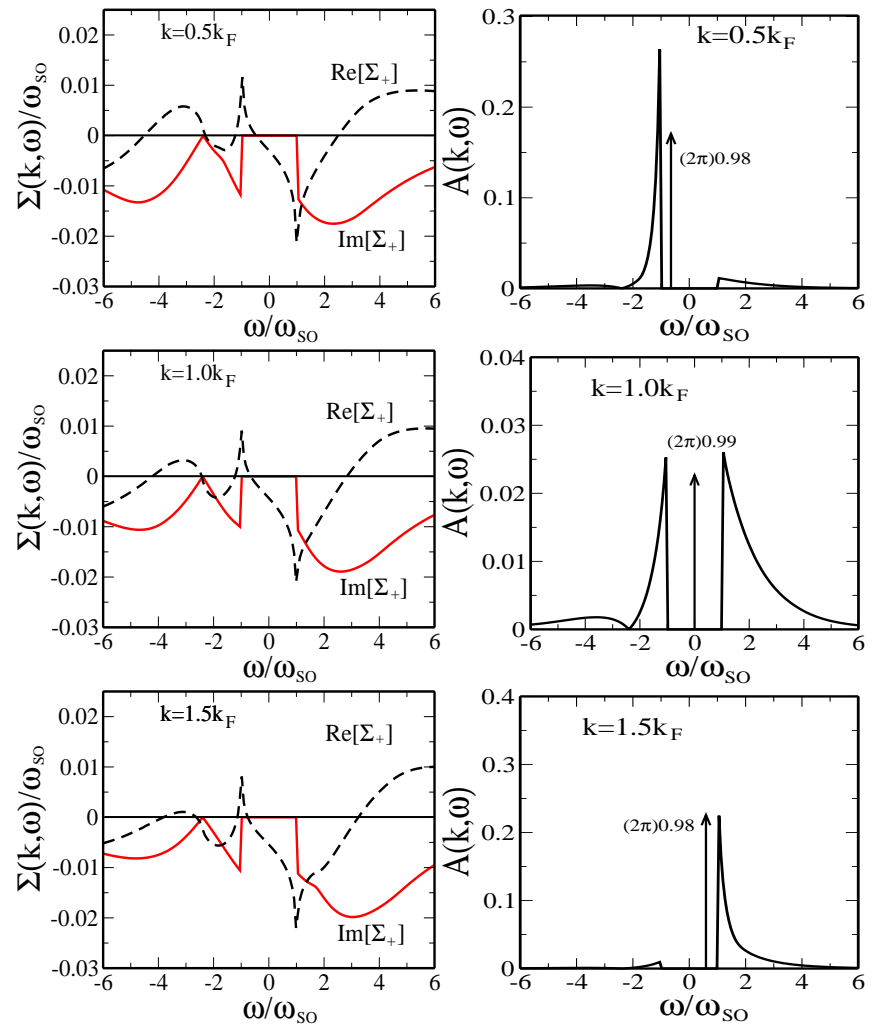

FIG. 5. Calculated self energy $\Sigma_{+}$and spectral function $A_{+}$ as a function of energy for different wave vectors, $k=0.5,1.0$, $1.5 k_{F}$ and for $d=1 \mathrm{~nm}$ and $T=0$. RPA screening is considered in the electron-SO phonon interaction. Arrows indicate $\delta$-function peak at the quasiparticle energy. The renormalized velocity due to the screened electron-SO phonon interaction is bareley reduced, giving $v_{F}^{*}=0.98 v_{F}$ approximately for these results.

spectively, and these quasiparticle peaks consume almost all $(99 \%)$ of the total spectral weight. Just less than $1 \%$ of the spectral weight goes to the incoherent background. The renormalized velocity due to the screened electron-SO phonon interaction is barely reduced, giving $v_{F}^{*}=0.98 v_{F}$ approximately for these results. Since screening reduces the magnitude of the imaginary part of the self energy as shown in Figs. 1 and 2 the spectral weight of the incoherent background is diminished in the presence of screening, which gives rise to the increase of the spectral weight in the quasiparticle peak because of the sum rule. In Fig. 6 we show the self-energy $\Sigma_{-}(k, \omega)$ and the spectral function $A-(k, \omega)$ calculated with the screened electron-SO phonon interaction. For $s=-1$ the quasiparticle peaks in the presence of screening are also incoherent and the peaks are broadened by emitting or absorbing SO phonons. However, the reduction of the magnitude of the self-energy due to screening the broadened quasiparticle peaks become much sharper than the peaks without screening.

In Fig. 7 we show the imaginary part of the on-shell self energy, i.e., the self energy at $\omega=\xi_{k}=v_{F} k-E_{F}$.
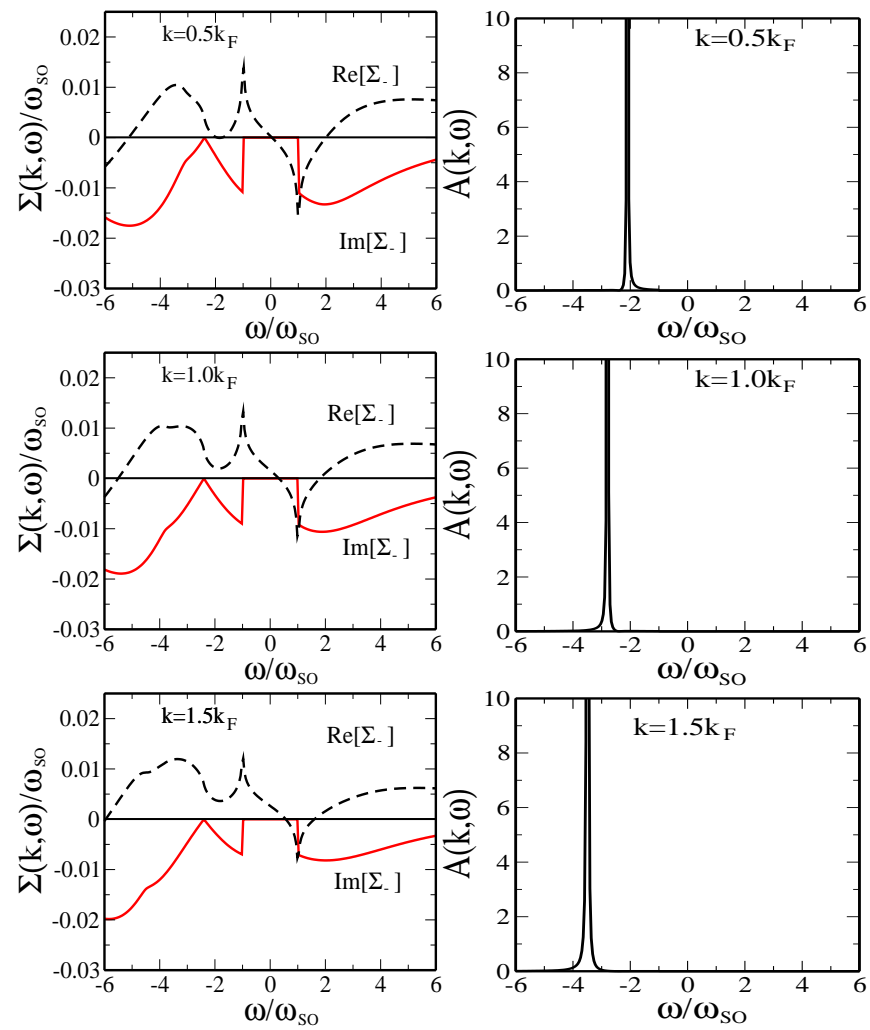

FIG. 6. Calculated self energy $\Sigma_{-}$and spectral function $A_{-}$ as a function of energy for different wave vectors, $k=0.5$, $1.0,1.5 k_{F}$ and for $d=1 \mathrm{~nm}$ and $T=0$. RPA screening effect is considered in the electron-SO phonon interaction.

For $0<k<k_{F}-\omega_{S O} / v_{F}$ electrons decay by absorbing SO phonons, while for $k>k_{F}+\omega_{S O} / v_{F}$ electrons decay by emitting SO phonons. Thus, $\operatorname{Im}\left[\Sigma_{s}\left(k, \xi_{k}\right)\right]=0$ for $k_{F}-\omega_{S O} / v_{F}<k<k_{F}+\omega_{S O} / v_{F}$. Inside the forbidden gap, the electron does not decay by emitting or absorbing a SO phonon. For $d=0$ and large wave vectors $k$ we have $\operatorname{Im}\left[\Sigma_{+}\left(k, \xi_{k}\right)\right] \propto G_{++}\left(k, k-\omega_{S O}\right)\left(k-\omega_{S O}\right) \propto \log (k)$ and $\operatorname{Im}\left[\Sigma_{-}\left(k, \xi_{k}\right)\right] \propto G_{-+}\left(k, k-\omega_{S O}\right)\left(k-\omega_{S O}\right) \sim 2$. The $\operatorname{Im}\left[\Sigma_{+}\left(k, \xi_{k}\right)\right.$ increases logarithmically and $\operatorname{Im}\left[\Sigma_{-}\left(k, \xi_{k}\right)\right.$ saturates for large $k$. Thus, the damping of electrons by emitting SO phonon depends weakly on the energy of electrons. However, the $\operatorname{Im}\left[\Sigma_{+}\left(k, \xi_{k}\right)\right]$ for the non-polar LO phonon increases linearly with wave vector and high energy electrons decay effectively by emitting more LO phonons 22,25 . Screening effects reduce the magnitude of the on-shell energy as shown in Fig. 7(c) and (d). In addition to the reduction due to screening the magnitude of the imaginary part of the on-shell self-energy decreases with increasing $d$ (the distance between graphene and the interface of the dielectric substrate).

In Fig. 8 the calculated damping rate, $\Gamma(k)=$ $2 \operatorname{Im}\left[\Sigma\left(k, \xi_{k}\right)\right]$, is shown as a function of wave vector for different temperatures. Here, we assume that the temperature of electron and phonon is the same. As temperature increases the forbidden gap disappears due to the thermal smearing of the Fermi surface, which 

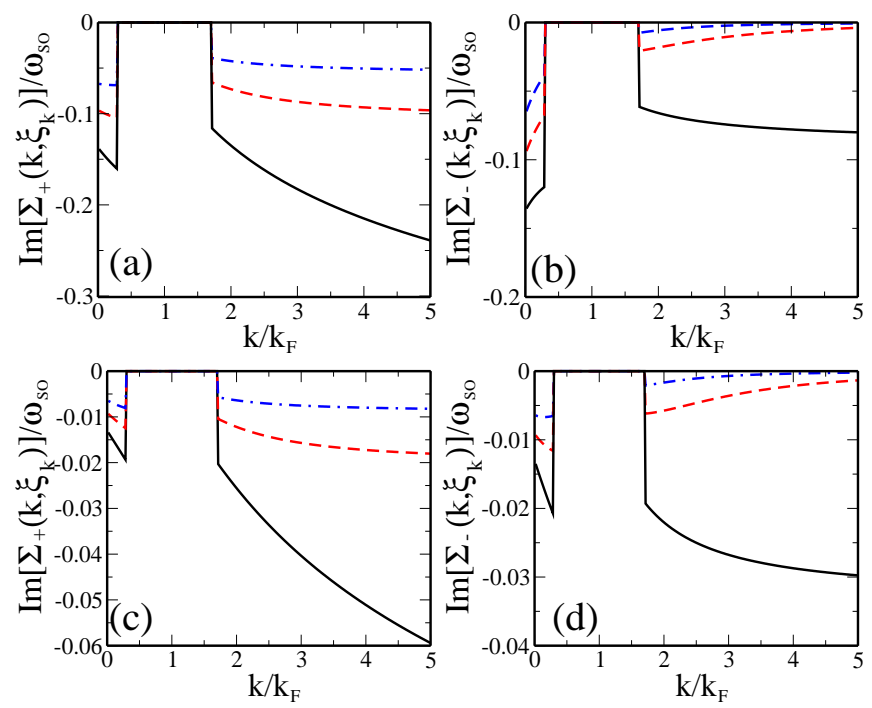

FIG. 7. The imaginary part of the on-shell self energy, $\operatorname{Im}\left[\Sigma\left(k, \xi_{k}\right)\right]$, as a function of wave vector for different $d=0$ (solid lines), 1 (dashed lines), $2 \mathrm{~nm}$ (dot dashed lines). The calculation is done at $T=0$. (a) and (b) are the results with unscreened electron-phonon interaction for $s=+1$ and $s=-1$, respectively. (c) and (d) are results with screening interaction for $s=+1$ and $s=-1$, respectively. The forbidden gap, where $\operatorname{Im}\left[\Sigma_{s}\left(k, \xi_{k}\right)=0\right]$, is given by $k_{F}-\omega_{S O} / v_{F}<$ $k<k_{F}+\omega_{S O} / v_{F}$.

loosens the phase space restriction. Thus, SO phonon emission (absorption) is allowed for $k<k_{F}+\omega_{S O} / v_{F}$ $\left(k>k_{F}-\omega_{S O} / v_{F}\right)$. The quantitative effect of screening (compared with the unscreened result) is about a factor of five reduction of the scattering rate as shown in Fig. 8 (c) and (d). In addition to the reduction of the rate, screening effects are particularly important in $\Gamma_{-}(k)$. In Fig. 8 (b) the peak inside the forbidden region at $T=0$ develops as temperature increases. The peak occurs at $k=\omega_{S O} / 2 v_{F}$ and arises from the singular nature of the long range bare electron-phonon interaction. The divergence of the interaction at $q=0$ disappears in the presence of screening effects and the developed peak in scattering rate also disappears due to screening effects.

In Fig. 9 the calculated damping rate at $k=k_{F}, \Gamma\left(k_{F}\right)$, is shown as a function of temperature for different electron densities. The results in Fig. 9 are calculated without screening effects, but we notice that the screening effects reduce the scattering rate approximately by a factor of five as shown in Fig. 9, The scattering rate increases exponentially with temperature due to the increases of phonon population, $\Gamma \propto e^{-\omega_{S O} / T}$ as temperature increases. Insets in Fig. 9] show the calculated damping rate as a function of density at $T=300 \mathrm{~K}$. The density dependent damping rate at a given temperature is non-monotonic and has a local minimum at the density corresponding to $E_{F}=\omega_{S O}$. Thus, the damping rate decreases first as the density increases, and has a minimum at $n=\left(\omega_{L O} / v_{F}\right)^{2} / \pi$. The damping rate increases as
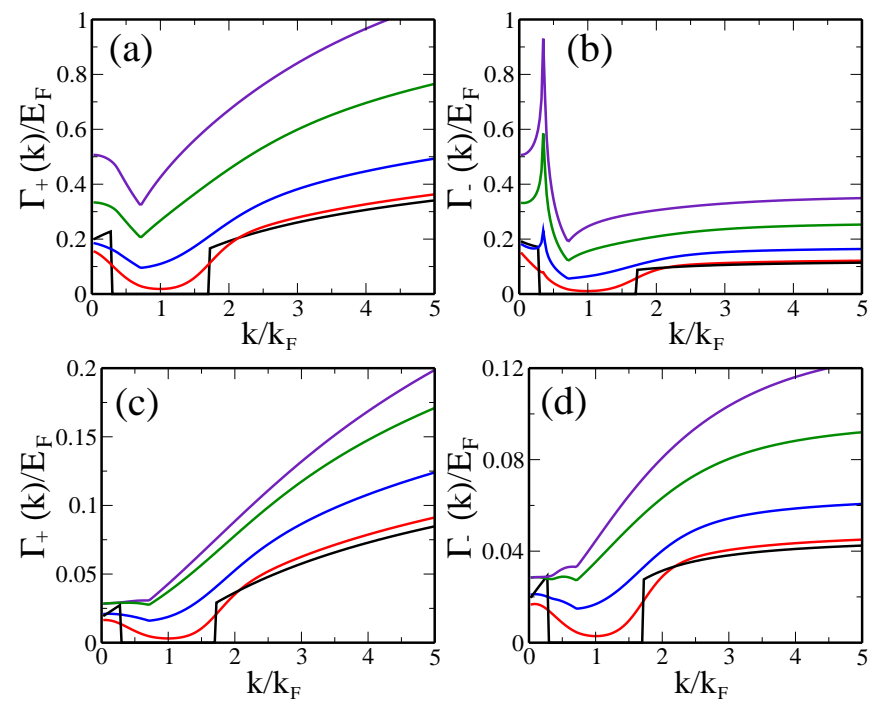

FIG. 8. Calculated damping rate $\Gamma(k)=2 \operatorname{Im}\left[\Sigma\left(k, \xi_{k}\right)\right]$ as a function of wave vector for different temperatures, $T=0,0.2$, 0.4, 0.7, 1.0 $T_{F}$ (from bottom to top), where $T_{F}=E_{F} / k_{B}$ is the Fermi temperature. The carrier density $n=2 \times 10^{12} \mathrm{~cm}^{-2}$ and $d=0$ are used in this calculation. (a) and (b) are the results with unscreened electron-phonon interaction for $s=$ +1 and $s=-1$, respectively. (c) and (d) are results with screened interaction for $s=+1$ and $s=-1$, respectively.

the density increases further. For $s=-1$ the damping rate shows more complicated density dependence. The local minimum occurs at the same density for $s=+1$, but there is a sharp peak at low density. The sharp peak in $\Gamma_{-}\left(k_{F}\right)$ arises from the unscreened electron-phonon interaction, and disappears when we consider the screening effects.

When the scattering rate $\Gamma$ is known, the conductivity can be calculated by using Boltzmann transport equation 2,7

$$
\sigma=\frac{e^{2} v_{F}^{2}}{2} \int d \omega \frac{D(\omega)}{\Gamma(\omega)}\left(-\frac{\partial f}{\partial \omega}\right)
$$

where $D(\omega)=g_{s} g_{v} \omega /\left(2 \pi v_{F}^{2}\right)$ is the density of states of graphene and $f$ is the Fermi distribution function. In Fig. 10 we show the calculated resistivity $\left(\rho=\sigma^{-1}\right)$ as a function of temperature for different $d$, the distance between the interface of the substrate and graphene. From the calculated resistivity the mobility is calculated by the simple formula $\mu=6.24 \times 10^{5} / \tilde{n} \rho \mathrm{cm}^{2} / \mathrm{Vs}$ where $\tilde{n}$ is the carrier density measured in unit of $10^{12} \mathrm{~cm}^{-2}$ and $\rho$ in unit of $\Omega$. The calculated resistivity limited by the electron-SO phonon scattering shows the exponential temperature dependence, which is the characteristic behavior of the optical phonon scattering arising from the thermal occupation factor in the scattering rate. The exponential temperature dependence is not affected by the screening effects as shown in Fig. 10(b). In insets of Fig. 10 we show the calculated conductivity as a function of density for different $d$. The density dependent 

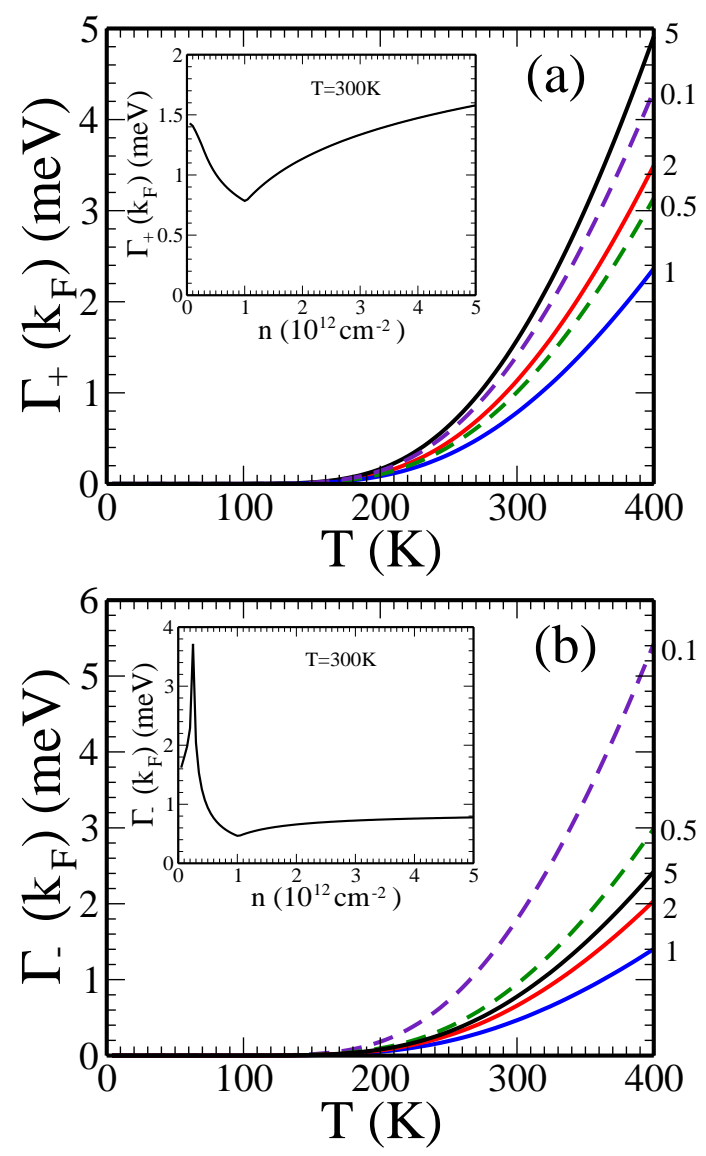

FIG. 9. Calculated damping rate at $k=k_{F}$ as a function of temperature for different electron densities, which are shown next to the lines in the unit of $10^{12} \mathrm{~cm}^{-2}$. The results are calculated with $d=0$ and without screening effects. Insets show the calculated damping rate as a function of density at $T=300 \mathrm{~K}$. The density dependent damping rate have a local minimum at the density corresponding to $E_{F}=\omega_{S O}$.

conductivity shows linear behavior, but the slopes are different for low and high density regions. The characteristic electron density $n_{c}$ separating these two regions is given by the density corresponding to the Fermi energy $E_{F} \sim \omega_{S O}$. Below $n_{c}$ the conductivity increases fast but above $n_{c}$ the conductivity increases slowly. The exponential temperature dependence of the resistivity and the linear density dependence of the conductivity have recently been observed in the transport measurement $\stackrel{5.6}{ }$.

\section{CONCLUSION}

We theoretically study various aspects of the electronsurface optical phonon interaction effects in graphene on a substrate made of polar materials. We provide a rather complete set of numerical results of self-energy, spectral function, and damping rate both for the unscreened interaction and for the static RPA screening interaction.

We find that the scattering rate with screened electron-
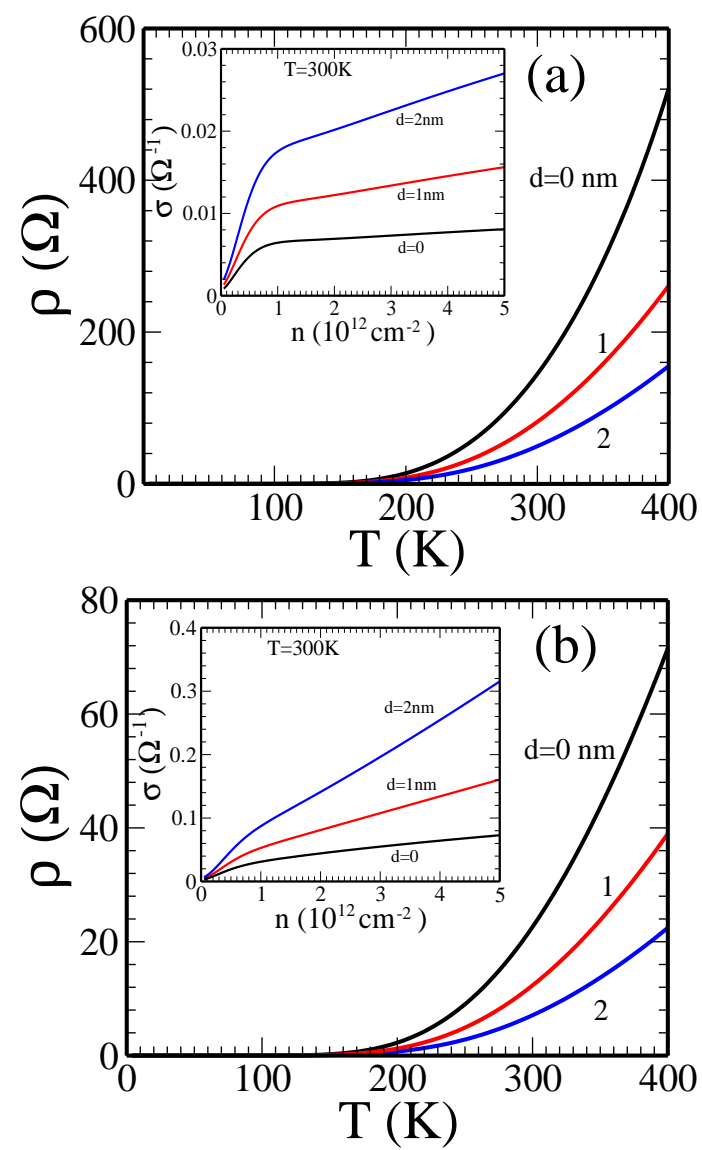

FIG. 10. Calculated resistivity $\rho=\sigma^{-1}$ as a function of temperature for different $d$, the distance between the dielectric interface and graphene. The carrier density $n=2 \times 10^{12}$ $\mathrm{cm}^{-2}$ is used in this calculation. In insets conductivity as a function of density for different $d$ is shown. In (a) the screening effects is not considered, but in (b) the RPA static screening is used. The mobility is calculated by the simple formula $\mu=6.24 \times 10^{5} / \tilde{n} \rho \mathrm{cm}^{2} / \mathrm{Vs}$ where $\tilde{n}$ is the carrier density $\left(\tilde{n}=n / 10^{12} \mathrm{~cm}^{-2}\right)$ and $\rho$ in unit of $\Omega$.

SO phonon interaction is reduced by a factor of five and the self-energy by a factor of ten, compared with the results with unscreened interaction. The static RPA screening used in this calculation may overestimate the screening effect on scattering rate, but we believe that the static RPA screening provides an upper limit of the screening effects. That is, our unscreened and statically screened results for the surface polar optical phonon scattering rate provide two extreme bounds for the magnitude of the scattering rate since static screening is an overestimation of the actual dynamical screening. We mention that the static screening approximation becomes very accurate at high carrier density when the typical Fermi energy exceeds the SO phonon energy, which may already happen at a rather low carrier density of $10^{12} \mathrm{~cm}^{-2}$. We therefore believe that our statically screened results should be valid for most graphene samples used experimentally. In addition to the reduction of 
the magnitude of the self-energy the screening effects remove the unphysical singular behavior in the self-energy for $s=-1$ arising from the long range nature of the electron-SO phonon interaction.

We find that the calculated renormalized Fermi velocity due to the unscreened electron-SO phonon interaction is reduced (with respect to the bare graphene velocity) by about $20 \%, v_{F}^{*} \sim 0.8 v_{F}$, but for the screened interaction it is reduced by only $2 \%, v_{F}^{*} \sim 0.98 v_{F}$ at a density $n=2 \times 10^{12} \mathrm{~cm}^{-2}$. We note that the renormalized Fermi velocity is enhanced about $20 \%$ at the same density when the electron-electron Coulomb interaction is only considered ${ }^{26}$. Since our consideration of screening in this paper is the RPA static dielectric function we cannot state conclusively that the small change of the renormalized velocity in the presence of screened electronphonon interaction arises from the direct cancellation between these two interactions. More careful calculation is need to confirm this cancellation by considering the dynamical screening effects treating both the electron-SO phonon and the electron-electron Coulomb interaction on an equal footing. But it appears the graphene velocity renormalization due to the electron SO phonon interaction by itself is a rather small quantitative effect because of screening effect. This is certainly true at high carrier densities $\left(>10^{12} \mathrm{~cm}^{-2}\right)$ where our static screening approximation should apply well. We also find that the scattering rate for the electron-SO phonon interaction depends weakly on the electron energy due to the cancellation between the long range nature of the interaction and the linear behavior of the density of states of graphene. For the in-plane non-polar LO phonon of graphene the scattering rate strongly depends on the electron energy and high energy electrons are heavily damped by emitting LO phonons. Since the scattering rate due to the SO phonon decreases further due to the physical separation between graphene and interface of the dielectric substrate the chance of the hot electron damping by emitting SO phonons may be very low in the real graphene samples, but the high-temperature transport in clean graphene may very well be dominated by SO phonon scattering from the substrate.

\section{ACKNOWLEDGMENTS}

This work was supported by U.S.-ONR.
1 T. Ando, A. B. Fowler, and F. Stern, Rev. Mod. Phys. 54, 437 (1982).

2 S. Das Sarma, S. Adam, E. H. Hwang, and E. Rossi, Rev. Mod. Phys. bf 83, 407 (2011).

3 G.D. Mahan, Many Particle Physics, 3rd ed. (Kluwer/Plenum, New York, 2000).

4 S. V. Morozov, K. S. Novoselov, M. I. Katsnelson, F. Schedin, D. C. Elias, J. A. Jaszczak, and A. K. Geim, Phys. Rev. Lett. 100, 016602 (2008)

5 J. H. Chen, C. Jang, S. Xiao, M. Ishigami, and M. S. Fuhrer, Nat. Nanotechnol. 3, 206 (2008).

${ }^{6}$ K. Zou, X. Hong, D. Keefer, and J. Zhu, Phys. Rev. Lett. 105126601 (2010); X. Hong, K. Zou, A. M. DaSilva, C. H. Ahn, and J. Zhu, Solid State Commun. 152, 1365 (2012).

7 S. Fratini and F. Guinea, Phys. Rev. B 77, 195415 (2008); J. Schiefele, F. Sols, and F. Guinea, Phys. Rev. B 85, 195420 (2012); X. Li, E. A. Barry, J. M. Zavada, M. B. Nardelli, and K. W. Kim, Appl. Phys. Lett. 97, 232105 (2010).

8 E. H. Hwang and S. Das Sarma, Phys. Rev. B 77, 115449 (2008).

9 Hongki Min, E. H. Hwang, and S. Das Sarma, Phys. Rev. B 83, 161404(R) (2011).

10 Dmitri K. Efetov and Philip Kim, Phys. Rev. Lett. 105, 256805 (2010); A. Pachoud1, M. Jaiswal, P. K. Ang, K. P. Loh, and B. Özyilmaz, Europhys. Lett. 92, 27001 (2010).

11 X. Du, I. Skachko, A. Barker, and E. Y. Andrei, Nat. Nanotechnol. 3, 491 (2008); K. I. Bolotin, K. J. Sikes, Z. Jiang, M. Klima, G. Fudenberg, J. Hone, P. Kim, and H. L. Stormer, Solid State Commun. 146, 351 (2008); A. S. Mayorov, D. C. Elias, I. S. Mukhin, S. V. Morozov, L. A. Ponomarenko, K. S. Novoselov, A. K. Geim, and R. V.
Gorbachev, Nano Lett. 12, 4629 (2012).

12 S. Das Sarma and E. H. Hwang, Phys. Rev. B 87, 035415 (2013); S. Adam and S. Das Sarma, Solid State Commun. 146, 356 (2008)

13 R. Fuchs and K. L. Kliewer, Phys. Rev. 140, A2076 (1965); K. L. Kliewer and R. Fuchs, ibid. 144, 495 (1966); R. Fuchs and K. L. Kliewer, ibid. 150, 573 (1966).

14 E. N. Economou, Phys. Rev. 182, 539 (1969).

15 S. Q. Wang and G. D. Mahan, Phys. Rev. B 6, 4517 (1972).

16 K. Hess and P. Vogl, Solid State Commun. 30, 807 (1979); M. V. Fischetti, D. A. Neumayer, and E. A. Cartier, J. Appl. Phys. 90, 4587 (2001).

17 Y. Liu and R. F. Willis, Phys. Rev. B 81, 081406 (2010).

18 J. Lu, K. P. Loh, H. Huang, W. Chen, and A. T. S. Wee, Phys. Rev. B 80, 113410 (2009).

19 R. J. Koch, Th. Seyller, and J. A. Schaefer, Phys. Rev. B 82, 201413(R) (2010)

${ }^{20}$ H. Yan, T. Low, W. Zhu, Y. Wu, M. Freitag, X. Li, F. Guinea, P. Avouris, F. Xia, arXiv:1209.1984.

21 E. H. Hwang, R. Sensarma, and S. Das Sarma. Phys. Rev. B 82, 195406 (2010).

22 W. -K. Tse and S. Das Sarma, Phys. Rev. Lett. 99, 236802 (2007).

23 C. -H. Park, F. Giustino, M. L. Cohen, and S. G. Louie, Phys. Rev. Lett. 99, 086804 (2007).

24 E. H. Hwang and S. Das Sarma, Phys. Rev. B 75, 205418 (2007).

25 M. Calandra and F. Mauri, Phys. Rev. B 76, 205411 (2007).

26 S. Das Sarma and E. H. Hwang, Phys. Rev. B 87, 045425 (2013) 